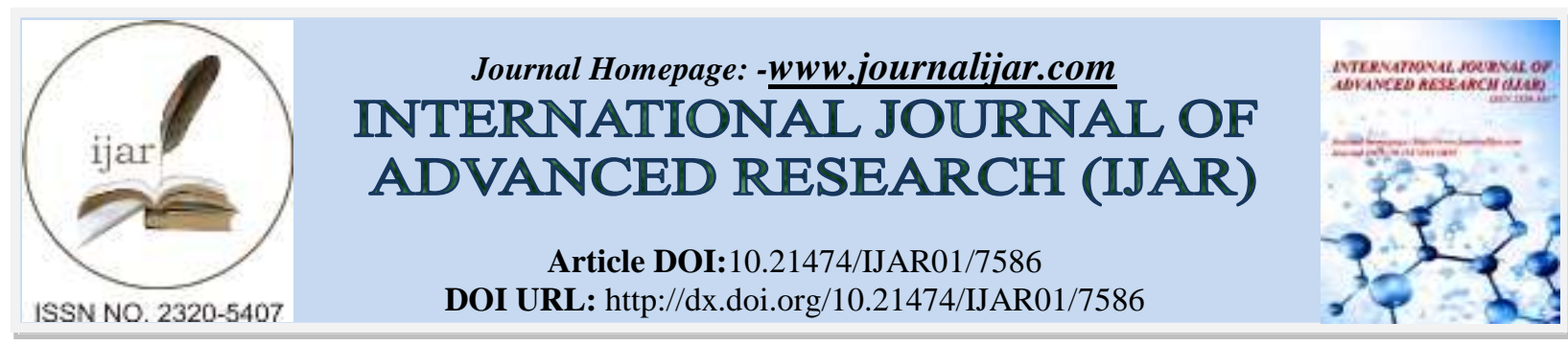

RESEARCH ARTICLE

\title{
MELIOIDOSIS - A CASE SERIES FROM DAKSHIN KANNADA.
}

1. junior resident $\mathrm{KS}$ hedge medical college.

Dr. Perumal Boney ${ }^{1}$ and Dr. Lancelot Lobo ${ }^{2}$.

2. Associate Professor K.S Hegde Medical college Mangalore, Department of General Surgery.

Manuscript Info

Manuscript History

Received: 17 June 2018

Final Accepted: 19 July 2018

Published: August 2018

\section{Abstract}

Copy Right, IJAR, 2018,. All rights reserved.

\section{Introduction:-}

Melidiosis is an infectious disease caused by gram negative organism Burkholderia pseudomallei(1). B.pseudomallei often described as soil dwelling organism resembling safety pin appearence on microbiological appearance. Melidiosis is endemic in South east Asia and Australia (2). Risk Factors have been associated are Diabetes, alcohol abuse, chronic kidney disease and chronic lung disease (3).In this case series of melidiosis from 2015-16 encountered in tertiary hospital.We emphasise on treatment modality.

\section{Case Series:-}

A 70 year old male presented with increased frequency of micturation and reddish Lesion over the chest since 10 days. He was known diabetic on Oral anti-diabetic treatment. CT Thorax showed well defined Loculated fluid collection in the anterior chest wall and around the sternum in the sub-cutaneous and intramuscular plane extending to the retro-sternal space. Similar pocket of fluid collection also seen in the left posterior extra pleural space with enhancing adjacent pleura with the larger collection extending in the intra muscular plane medially into the spinal canal through neural foramina. Multiple cavitating lesion in both lungs with minimal bilateral pleural effusions also noted. Aspirate from the sub-cutaneous swelling grew Burkholderia psudomaleli. He was treated with Injection Meropenem for 14 days followed by T. Septran DS 1-0-1 for 4 weeks. At 6 month follow up patient had no recurrence.

A 55 year female presented with fever, headache and loose stools for 20 days. Patient is Known Diabetic on treatment. USG showed No Organomegaly, Minimal ascites and minimal left pleural effusion. Blood culture showed Burkholderia psudomallei. Sputum AFB was negative. Patient was treated with injection Meropenem $1 \mathrm{gm}$ for 14 days and followed by T. Septran DS for 4 weeks. At 6 month follow up patient had no recurrence.

A 62 year male was admitted with complaints of low back pain and fever since 3 weeks. History of loss of weight and appetite for last 6 months. Patient was known case of COPD on medication and diabetes on oral hypoglycaemic agents for last 10 years. CT abdomen showed Gross splenomegaly and multiple hypodense area peri-splenic collection with pleural effusion and loculated pleural space collection. Pleural tapping was done showed exudative 
effusion. Pleural fluid grew Burkholderia psudomalleli. Patient was treated with Injection meropenem for 14 days. Patient was started on T. Septran DS and Doxycycline for 4 weeks. Patient had no recurrence after 6month.

\subsection{Case 4}

A 63 Year female presented with complaints of fever, burning micturation, breathlessness and decreased urine output for 15 days. Patient was known diabetic on treatment. Patient had B/L Pneumonia and urinary tract infection. Blood culture showed Burkholderia pseudomallei . Patient was started on Injection Meropenem for 14 days and T. Doxycycline 100mg 1-0-1 for 6 days. Patient had no recurrence after 2 month follow up.

\section{Case 5:-}

A 20 year female came with complaints of fever with chills and cough with scanty expectoration for last 8 days. Patient is known case of type 1Diabetes Mellitus from 12 year of age. She was diagnosed with bronchopneumonia with right sided pneumothorax. Patient had ICD place and serial culture were sent Burkholderia pseudomalleli grew and antibiotics was started Injection Meropenem for 14 days. patient was discharged against medical advise.

\section{Case 6:-}

A 46 year male was admitted with complaints of fever with chills cough with expectoration since 2 weeks and yellowish discolouration of eye for last 3 days. Patient is case of Right Tuberculosis arthritis completed DOTS I and Diabetes mellitus on medication. Ct chest showed Mild Hepatospleenomegaly and multiple randomly distributed heterogeneously enhancing nodules with irregular margins in B/L lungs with mediastinal Lymph nodes. Patient was started on Injection Ceftazidime and meropenem for 14 days. Patient was started on T. Bactrum DS for 3 months and doxycyclineline for 3 months. Patient had no recurrence after 3 month follow up.

\section{Material and Method:-}

Our centre is a tertiary care referral hospital to which patients from many parts of karnataka especially dakshin kannada and northern kerala in India are referred. Cases were patients with culture proven melioidosis who were admitted between January 2015 and December 2016. Ours was a retrospective study total of 6 patient were admitted. The patient records were reviewed with respect to age, gender and sex.

\section{Discussion:-}

The pathologist Alfred Whitmore and C.S. Krishnaswami explained meliodosis as a "glandular-like" disease among morphia addicts in Rangoon, Burma, in 1911(4).

Our case series is from karnataka with no specific age group youngest being 20 year to 70 year. In our study men and women were affected equally. In this case series all patient affected with melidiosis were known diabetic or newly diagnosed diabetic. Diabetes and alcohol abuse are proven risk factor for melidiosis (3). All of our patient were from rural areas and most of them had exposure to soil and water in the field areas.

In our case series all patient presented with fever as the most common complaints. 5 patient had bronchopneumonia of which 2 patient had lung nodule, and 1 patient had septic tubercular arthritis and had completed DOTS.

Most of our patient were started on combination of Injection ceftazidime in early phase and responded well to the treatment(5).Clinical presentations of melioidosis has been described as the fulminant end of the clinical spectrum, with abscesses throughout both lungs, and in many organs(6). At the other end of the spectrum are asymptomatic infections and localized skin ulcers or abscesses without systemic illness. Howe and colleagues have classified melioidosis as acute, sub-acute and chronic (7). On chest radiographs, diffuse nodular infiltrates often develop throughout both lungs and they coalesce, cavitate, and progress rapidly, consistent with caseous necrosis and multiple metastatic abscess formation seen at autopsy (8). Before, 1989, conventional therapy for melioidosis consisted of a combination of chloramphenicol, sulfamethoxazole-tromethoprim, doxycycline and sometimes kanamycin, given for 6 weeks to 6 months (9).

The actual cause for the higher incidence of melioidosis in india remain unclear. We assume in our study higher incidence rate was diagnosis in all patients with severe sepsis, abscess, septic arthritis and severe pneumonia. Diabetes has increased in the indian population and plays important role in increased incidence of the disease and most of the patient are from rural backgrounds who have exposure to the soil and water . 


\section{Conclusion:-}

Melioidosis is a emerging infectious disease in india which is treatable. It needs to be considered as one of the differential diagnosis in patient presenting with sepsis syndromes and focal abscesses, mostly in diabetics. Isolation of B. pseudomallei from body fluids of patients remains the "gold standard" in diagnosis and requires the use of selective media for non-sterile specimens. Gram stain and other histopathological stains are not specific for the organism. Medical therapy ceftazidime. Followed by cotrimoxazole for 12 weeks and has shown excellent response.

\section{References:-}

1. Whitmore A 1913. An account of a glanders-like disease occurring in Rangoon. J. Hyg. 13:1-34.

2. Muhammad ra Hassan, Subhada p pani, et al. incidence, risk factors and clinical epidemiology of melioidosis: a complex socio- ecological emerging infectious disease in the alor setar region of Kedah, malaysia. BMC Infectious Diseases 2010;10:302.

3. Currie BJ, Fisher DA, Howard DM, et al. The epidemiology of melioidosis in Australia and Papua New Guinea. ActaTrop.2004;74:121-127.

4. Whitmore A,Krishnaswami CS.An account of the discovery of a hitherto undescribed infective disease occurring among

5. population of Rangoon.Indian Med Gaz. 1912; 47: 262-67

6. .White, N. J., D. A. Dance, W. Chaowagul, Y. Wattanagoon, V. Wuthiekanun, and N. Pitakwatchara. 1989. Halving of mortality of severe melioidosis by ceftazidime. Lancet ii:697-701.

7. Mathew s, Perakath $b$, Mathew $G$ et al. surgical presentation of melioidosis in india. Natl med $J$ india 1999;12:59-61.

8. Curries BJ.Burkholderia pseudomallei and Burkholderia mallei:Melioidosis and Glanders.In:Mandel Douglas and Burnnett's

9. Principles and Practice of Infectious Diseases, 7th Ed.Mandell GLBennett JE,Dolin R(editors).Churchill Livingstone Elsevier,

10. 2010 .

11. .Howe C,Sampath A,Spotnitz M.The pseudomallei group:A review.J Infect Dis. 1971; 124:598-606.

12. K saravu, c mukhopadhyay, et al. melioidosis in southern india: Epidemiological and clinical pro le. Southeast Asian J Trop Med Public Health 2010;41:401-9.

13. Leelarasamee A, Bovornkitti S. Melioidosis: Review and update. Rev Infect Dis. 1989;11:413-425.

14. John $\mathrm{T} \mathrm{J}$, Jesudason $\mathrm{mv}$, lalitha $\mathrm{mK}$ et al. melioidosis in india: the tip of the iceberg? Indian $\mathrm{J}$ Med Res 1996;103:62-5.

15. Navaneethan u, Ramesh Kumar, Ravi G. multiple visceral abscess in a case of melioidosis. Indian J Medical Science 2006;60;68-70.

16. Mukhopadhya a, balaji V, Jesudason $\mathrm{mV}$, amte a, Jeyamani $\mathrm{r}$, Kurian $\mathrm{G}$. isolated liver abscesses in melioidosis. Indian J Med Microbiol 2007;25:150-1

17. Vidyalakshmi K, chakrapani $\mathrm{m}$, shrikala b, damodar s, lipika s, Vishal s. tuberculosis mimicked by melioidosis. Int J Tuberc Lung Dis 2008;12:1209-15. 